\title{
Eect of Si-doped InGaN underlayers on photoluminescence eciency and recombination dynamics in InGaN/GaN quantum wells
}

DOI:

10.1088/1361-6463/ac22d3

\section{Document Version}

Accepted author manuscript

Link to publication record in Manchester Research Explorer

Citation for published version (APA):

Church, S., Christian, G., Barrett, R., Hammersley, S., Kappers, M. J., Frentrup, M., Oliver, R. A., \& Binks, D. (2021). Eect of Si-doped InGaN underlayers on photoluminescence eciency and recombination dynamics in InGaN/GaN quantum wells. Journal of Physics D Applied Physics. https://doi.org/10.1088/1361-6463/ac22d3

Published in:

Journal of Physics D Applied Physics

\section{Citing this paper}

Please note that where the full-text provided on Manchester Research Explorer is the Author Accepted Manuscript or Proof version this may differ from the final Published version. If citing, it is advised that you check and use the publisher's definitive version.

\section{General rights}

Copyright and moral rights for the publications made accessible in the Research Explorer are retained by the authors and/or other copyright owners and it is a condition of accessing publications that users recognise and abide by the legal requirements associated with these rights.

\section{Takedown policy}

If you believe that this document breaches copyright please refer to the University of Manchester's Takedown Procedures [http://man.ac.uk/04Y6Bo] or contact uml.scholarlycommunications@manchester.ac.uk providing relevant details, so we can investigate your claim.

\section{OPEN ACCESS}




\title{
Effect of Si-doped InGaN underlayers on photoluminescence efficiency and recombination dynamics in InGaN/GaN quantum wells
}

\author{
S. A. Church, ${ }^{1}$ G. M. Christian, ${ }^{1}$ R. M. Barrett, ${ }^{1}$ S. Hammersley, ${ }^{2}$ M. J. Kappers, ${ }^{3}$ M. Frentrup, ${ }^{3}$ R. A. Oliver, ${ }^{3}$ \\ and D. J. Binks ${ }^{1}$ \\ ${ }^{1)}$ Department of Physics and Astronomy and Photon Science Institute, University of Manchester, \\ Manchester M13 9PL, United Kingdom \\ ${ }^{2)}$ Department of Electrical and Electronic Engineering and Photon Science Institute, University of Manchester, \\ Manchester M13 9PL, United Kingdom \\ ${ }^{3)}$ Department of Materials Science and Metallurgy, University of Cambridge, 27 Charles Babbage Road, \\ Cambridge CB3 OFS, United Kingdom
}

A series of single InGaN/GaN quantum wells with a Si-doped InGaN underlayer were studied to investigate the impact of the underlayer on photoluminescence efficiency and recombination dynamics. The thickness of the GaN capping layer was varied between samples, which changed the electric field across the QW due to band bending near the surface. When directly exciting the wells, thermionic emission of carriers results in a rapid drop in the photoluminesence efficiency with increasing temperature such that no emission is observed above $100 \mathrm{~K}$. However, exciting above the energy of the barriers caused the intensity of the QW emission to drop more slowly, with up to $12 \%$ of the $10 \mathrm{~K}$ emission intensity remaining at $300 \mathrm{~K}$. This difference is attributed to hole transfer from the underlayer into the quantum well, which increases in efficiency at higher temperatures, and is enhanced by stronger electric fields present in the GaN barriers of samples with thinner GaN capping layers. Further, the sample with the narrowest cap layer of $2 \mathrm{~nm}$ has a different shape and characteristic time for its photoluminescence decay transient and a different emission energy temperature dependence than the other samples. This behaviour was ascribed to a change in carrier localisation for this sample due to a reversal of the net field across the well compared to the other samples.

\section{INTRODUCTION}

The active regions of commercial white-light LEDs consist of a series of blue-emitting InGaN/GaN quantum wells (QWs). White light is achieved by means of a phosphor which absorbs a proportion of the blue light and emits yellow light. It has been shown that strong internal electric fields play an important role in the optical properties of these $\mathrm{QWs}^{1-3}$. The fields originate from the spontaneous and piezoelectric polarisation of the GaN crystal which, in conjunction with the strain in the QW, result in electric fields of the order of a few $\mathrm{MV} \mathrm{cm}{ }^{-1}{ }^{4}$. These fields are sufficient to overcome the Coulomb attraction between holes and electrons in the QWs, tending to push them to opposite QW interfaces and thereby reducing the wavefunction overlap of the carriers and so also reducing the radiative recombination rate $^{5}$. At elevated temperatures, when non-radiative recombination becomes competitive, this results in a reduction in the internal quantum efficiency (IQE). To achieve green emission, a higher indium content is required in the QW, which increases the strain and thus the electric field strength, thereby reducing the IQE further. This effect is believed to be an important contributor to the reduction in efficiency of longer wavelength emitting InGaN/GaN QWs, which is known as the "green gap"5,6.

Another important phenomenon in InGaN/GaN QWs is the localisation of carriers by inhomogeneities in the QW. Atomistic calculations have shown that electrons and holes are independently localised by QW width fluctuations and indium content fluctuations, respectively ${ }^{7}$. Furthermore, the hole localisation energies in these states can be larger than the thermal energy at room temperature $^{3}$. The high IQE of blue-emitting QWs (up to 95\%) is believed to be a consequence of this localisation, reducing the diffusion of carriers to non-radiative recombination sites ${ }^{7,8}$. The carrier localisation typically results in a characteristic "s-shape" temperature dependence of the peak emission energy in photoluminescence (PL) spectra $^{9}$, an extended PL decay time and non-exponential PL decay shape ${ }^{7,10}$.

Underlayers (ULs) are regions of unintentionally- or Si-doped (In) GaN that are typically $10-20 \mathrm{~nm}$ in thickness and positioned a few nm below the QWs. ULs are of interest because their incorporation into device structures has been found to improve the IQE of QWs ${ }^{11,12}$. Initial work on doped ULs found that they increase the radiative recombination rate by reducing the net electric field across the QWs ${ }^{11,12}$. This is a consequence of the band bending near the sample surface due to the pinning of the Fermi level by the doping of the UL and at the sample surface; the electric field resulting from this band bending counteracts the polarisation field such that the net field across the QW is reduced or even reversed, with the strength of the field due to the UL depending on its distance from the surface ${ }^{4}$. Other studies have shown that the UL reduces the non-radiative recombination rate in the $\mathrm{QW}$ at room temperature ${ }^{13}$. This has been attributed to the trapping of point defects in the UL, which thereby reduces the point defect density in the $\mathrm{QWs}^{14}$. Thus QWs can benefit from both the enhancement of the radiative recombination rate and the suppression of the non-radiative rate due to the UL.

Previous work has reported that the low temperature PL peak width is reduced for samples where the net field 
across the QW has been significantly reduced by the inclusion of an $\mathrm{UL}^{15}$. This behaviour was found to be consistent with an atomistic model that predicted that the electrons in samples with reduced net fields would be exposed to a different localisation landscape to the one experienced by electrons in samples without ULs. These different localisation landscapes correspond to a different range of recombination energies and hence PL peak widths. The model also found that whilst the average electric field across a QW could be zero, local fluctuations of the indium content resulted in variation in strain and therefore piezoelectric polarisation such that the net electric field was locally non-zero and, moreover, these local fields could have a similar magnitude to those in QWs without ULs. It is therefore expected that the recombination mechanism in QWs with ULs will differ not only from that occurring in polar QWs without ULs but also from that found in conventional non-polar QWs, such as those grown in the $m$-plane, even if the net field is approximately zero on average. It is important to understand these differences as they may have consequences for the IQE of QWs grown with a UL, along with QWs grown using other techniques to cancel out the fields. Similar structures to ULs have also been investigated as electron reservoir layers, designed to feed additional electrons into MQW structures ${ }^{16}$.

To investigate the effects of ULs further, we characterise in detail the optical properties of a series of single InGaN/GaN QWs grown with a Si:InGaN UL; each sample varies only in the thickness of its cap layer, which determines the field strength across the QW. We use temperature dependent PL to compare the IQE of the QWs when excited directly and indirectly, and this is combined with PL-excitation (PLE) spectroscopy to understand the process of carrier capture into the QW. Timeresolved $\mathrm{PL}$ measurements are also used to investigate the effect of the UL on the recombination dynamics.

\section{EXPERIMENTAL METHODS}

A series of four single QW structures were grown on $2 \mu \mathrm{m}$-thick GaN/sapphire templates using metalorganic chemical vapour deposition (MOCVD). On these templates a $24 \mathrm{~nm}$-thick and $2 \times 10^{19} \mathrm{~cm}^{-3}$ Si-doped InGaN UL with $5.9 \%$ InN-content, as well as a 3.5 nm-thick undoped GaN barrier were deposited prior to the growth of the single QWs. Previous work ${ }^{11,15}$ has shown that ULs with this In content do not significantly absorb the QW emission. The QWs are $2.7 \mathrm{~nm}$ thick, with an indium content of $18 \%$; further details of the sample growth are provided elsewhere ${ }^{15}$. The samples were designed to have an electric field strength close to zero across the QW. This was achieved by varying the thickness of the GaN cap layer to alter the surface band bending due to the Fermi level pinning at the UL. Cap thicknesses of $2 \mathrm{~nm}$, $3 \mathrm{~nm}, 4 \mathrm{~nm}$ and $5 \mathrm{~nm}$ were used, which were calculated to produce electric fields in the QW of $0.122 \mathrm{MV} \mathrm{cm}^{-1}$,
$0.0374 \mathrm{MV} \mathrm{cm}^{-1},-0.158 \mathrm{MV} \mathrm{cm}^{-1}$ and $-0.357 \mathrm{MV} \mathrm{cm}^{-1}$ respectively, using the 'nextnano3' device simulator ${ }^{15}$; these values are about an order of magnitude lower than for QWs without an $\mathrm{UL}^{4}$. Thus, the $3 \mathrm{~nm}$ cap sample is expected to have a close to zero average field, and the $2 \mathrm{~nm}$ cap sample a reversed field. Since the ULs are the same between all samples, any gettering of point defects observed in previous studies will also be the same in each sample ${ }^{14}$.

Temperature dependent PL was performed by mounting them on the cold finger of a close-cycled He cryostat, enabling sample temperatures between $10 \mathrm{~K}$ and $300 \mathrm{~K}$ to be achieved. Excitation was carried out using either a $325 \mathrm{~nm}$ continuous-wave HeCd laser, to achieve excitation above the GaN barriers, or a $405 \mathrm{~nm}$ laser diode, to excite the QWs directly. These lasers were focused onto the samples to achieve approximate incident power densities of $10 \mathrm{~W} \mathrm{~cm}^{-2}$ and $200 \mathrm{~W} \mathrm{~cm}^{-2}$, respectively, with the $325 \mathrm{~nm}$ light absorbed by the GaN, UL and QW and the $405 \mathrm{~nm}$ light absorbed just by the QW. The emission was focused onto the slit of a $0.85 \mathrm{~m}$ double grating spectrometer and detected using a GaAs photomultiplier tube with lock-in amplification. The sample was orientated at Brewster's angle which, when combined with a polariser, reduces the visiblity of Fabry-Perot interference on the spectrum $^{17}$. PLE spectroscopy was performed at $10 \mathrm{~K}$ using a Xe lamp coupled to a monochromator to create a variable wavelength excitation source with a power density of $0.4 \mathrm{~mW} \mathrm{~cm}^{-2}$.

Time-resolved PL was carried out with a $100 \mathrm{fs}$ pulsed Ti:sapphire laser, which was either frequency doubled to $400 \mathrm{~nm}$ or tripled to $266 \mathrm{~nm}$. The emitted photons were measured using a micro-channel plate with a $1 / \mathrm{e}$ time resolution of $70 \mathrm{ps}$, and time decays were produced using single photon counting techniques.

\section{RESULTS}

Fig. 1 shows the simulated conduction $(\mathrm{CB})$ and valence band (VB) profiles for the $3 \mathrm{~nm}$-capped QW sample, with and without an UL. With the UL present, the electric field across the QW is close to zero and the CB and VB in the QW (shaded grey) are approximately flat. The band edge profiles for the samples with other cap-thicknesses are shown in the supplementary material (S.M.).

The temperature dependence of the PL spectrum of the $2 \mathrm{~nm}$-capped QW is shown in Fig. 2. In this measurement, the excitation was above the bandgap of the GaN, and so, in addition to direct photon-absorption in the QW, electrons and holes are produced by photonabsorption in the GaN, and subsequently captured into the QW and the UL. In the low temperature spectra, the recombination of carriers in the UL results in an emission peak close to $3.19 \mathrm{eV}$ and recombination in the $\mathrm{QW}$ results in a peak close to $2.76 \mathrm{eV}$; the peak at an energy $91 \mathrm{meV}$ lower than the QW emission is the LO-phonon 


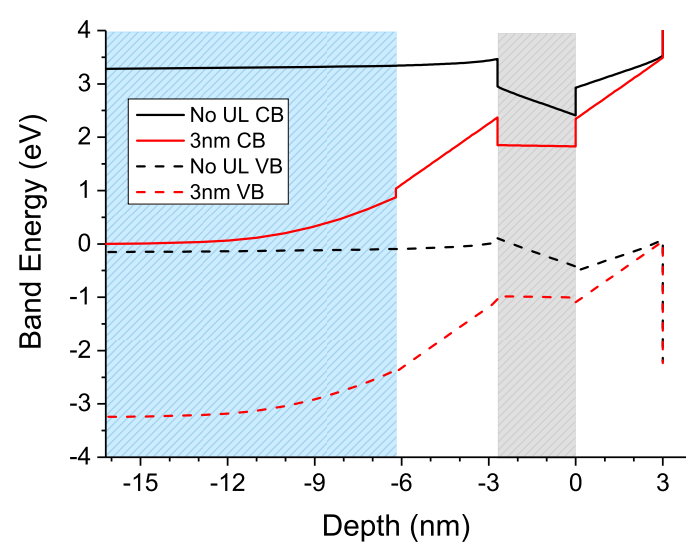

FIG. 1. The simulated CB and VB profiles for the $3 \mathrm{~nm}$ capped QW sample with and without the UL. The blue and grey shaded areas indicate the UL and QW regions, respectively. The zero of depth is taken as the upper surface of the QW, and energies are defined relative to the Fermi level.

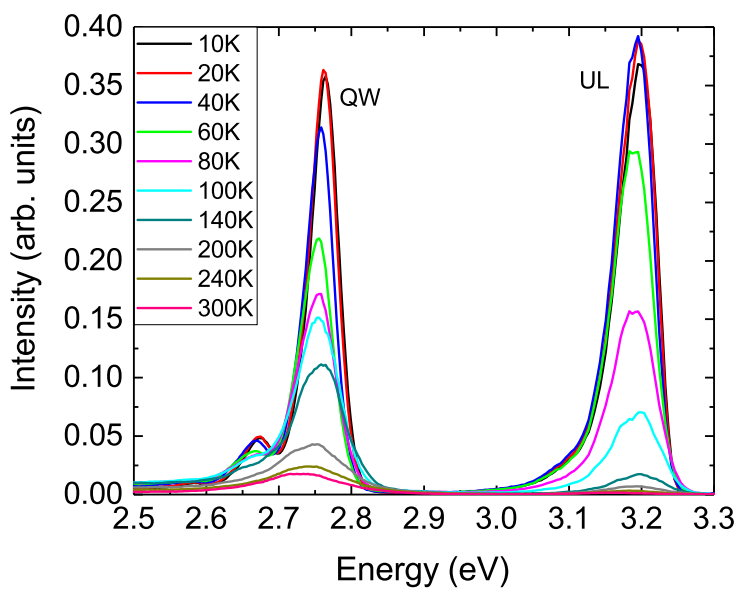

FIG. 2. Temperature dependent PL spectra for the $2 \mathrm{~nm}$ capped QW sample with excitation above the bandgap of the GaN. This spectrum shows emission from both the QW and the UL. The spectra are slightly modulated by Fabry-Perót interference fringes ${ }^{17}$.

replica of the QW peak ${ }^{17}$. These results are consistent with those previously reported ${ }^{15}$, and are similar for all the UL samples.

Fig. 3 shows the temperature-dependence of the spectrally-integrated emission due to direct excitation of the QW for the $2 \mathrm{~nm}$-capped sample, and of the $5 \mathrm{~nm}$ capped sample for comparison. In both cases, the intensity of the QW emission drops off rapidly above $10 \mathrm{~K}$, and no emission can be detected above $120 \mathrm{~K}$. This behaviour is typical for single QWs, where the holes escape the QW at elevated temperatures via thermionic emission $(\mathrm{TE})^{18}$. When the QWs are directly excited in this

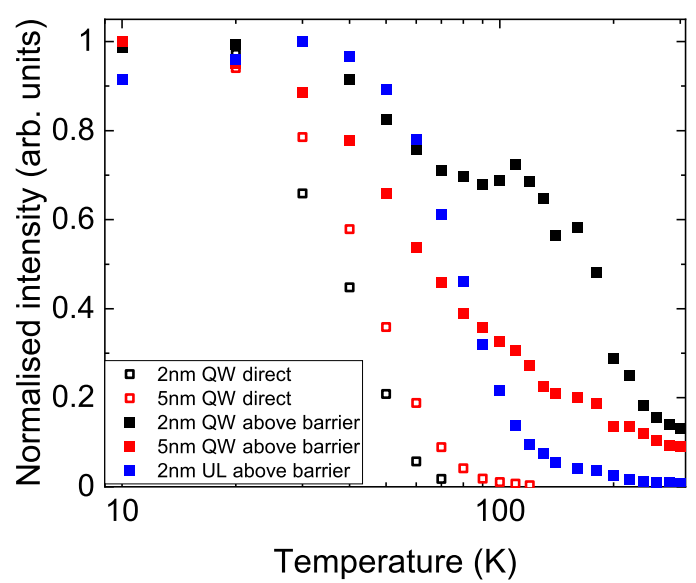

FIG. 3. The integrated intensity of emission peaks in PL spectra at different temperatures, normalised to the intensity at $10 \mathrm{~K}$ for each peak. Solid squares show results obtained with above barrier excitation, and open squares are with direct excitation of the QWs. Similar data for the other samples is given in the S.M.

way, no UL peak is observed, even at elevated temperatures when TE is occurring. This is consistent with the holes that escape the QW via TE being pushed away from the UL, towards the sample surface, by the strong electric fields in the QW barriers ${ }^{15}$.

However, as also shown in Fig. 3, quite different behaviour is observed for excitation above the barrier. Notably the intensity of the QW emission reduces much more slowly than when compared to the direct excitation experiment. Furthermore, the intensity appears to plateau for the $2 \mathrm{~nm}$-capped sample around $100 \mathrm{~K}$, before dropping at higher temperatures. This slowed decrease in the QW emission coincides with the decrease in the UL emission, which occurs for temperatures above $40 \mathrm{~K}$ as shown in Fig. 3. This behaviour is consistent with carrier transfer from the UL to the QW for temperatures above $40 \mathrm{~K}$.

The normalised integrated intensity is typically taken as an estimate of the relative IQE of the emission, assuming that the recombination is purely radiative at $10 \mathrm{~K}$. This indicates that the IQE of the QWs, when directly excited, drops to less than $1 \%$ at $100 \mathrm{~K}$ and is likely significantly smaller at room temperature, but is beyond the sensitivity of the experiment. When exciting above the barrier, however, the room temperature relative IQE is $12 \%$ and $9 \%$ for the $2 \mathrm{~nm}$ and $5 \mathrm{~nm}$-capped QWs.

Low temperature PL time decays were measured for above barrier excitation and the 1 /e decay times are shown in Fig.4. The 1/e decay times for the ULs are similar for each sample and vary between $0.3 \mathrm{~ns}$ and $1.0 \mathrm{~ns}$ across the emission peak. These lifetimes are close to recombination times in $\mathrm{GaN}^{19}$, as expected due to the relatively small indium content of the UL. The PL de- 



FIG. 4. $10 \mathrm{~K} 1 / \mathrm{e}$ decay times taken from PL time decays of QWs with different capping layer thicknesses and for above barrier excitation. The $10 \mathrm{~K} \mathrm{PL}$ spectrum for the $3 \mathrm{~nm}$-capped sample is provided for comparison. (a) Decay times across the QW emission. (b) Decay times across the UL emission.

cays for QW emission on a reduced timescale are also shown in Fig.5. The 1/e decay times are between $2 \mathrm{~ns}$ and $12 \mathrm{~ns}$ and increase at reduced energies. The decay shapes are also non-exponential. This behaviour is consistent with the recombination of independent electrons and holes in polar $c$-plane $\mathrm{QWs}^{10}$. The decay times flattens at low energies due to the spectra overlap with the phonon-replica. In comparison, the decay times for a non-polar QW are typically faster by an order of magnitude and have a mono-exponential shape, a consequence of excitonic recombination ${ }^{7}$. These results thus strongly suggest that the electric field is not zero across the QWs studied in this report. As discussed previously ${ }^{15}$, this is consistent with an electric field across the QWs that is close to zero on average, but varies locally due to indium content fluctuations with a magnitude that is similar to the average field across QWs without a UL.

As with the temperature dependent PL, the decay time results for the $2 \mathrm{~nm}$-cap sample are different from the others. At high emission energies the decay times are the same as the other samples, but they become longer than the other samples at lower energy. This difference

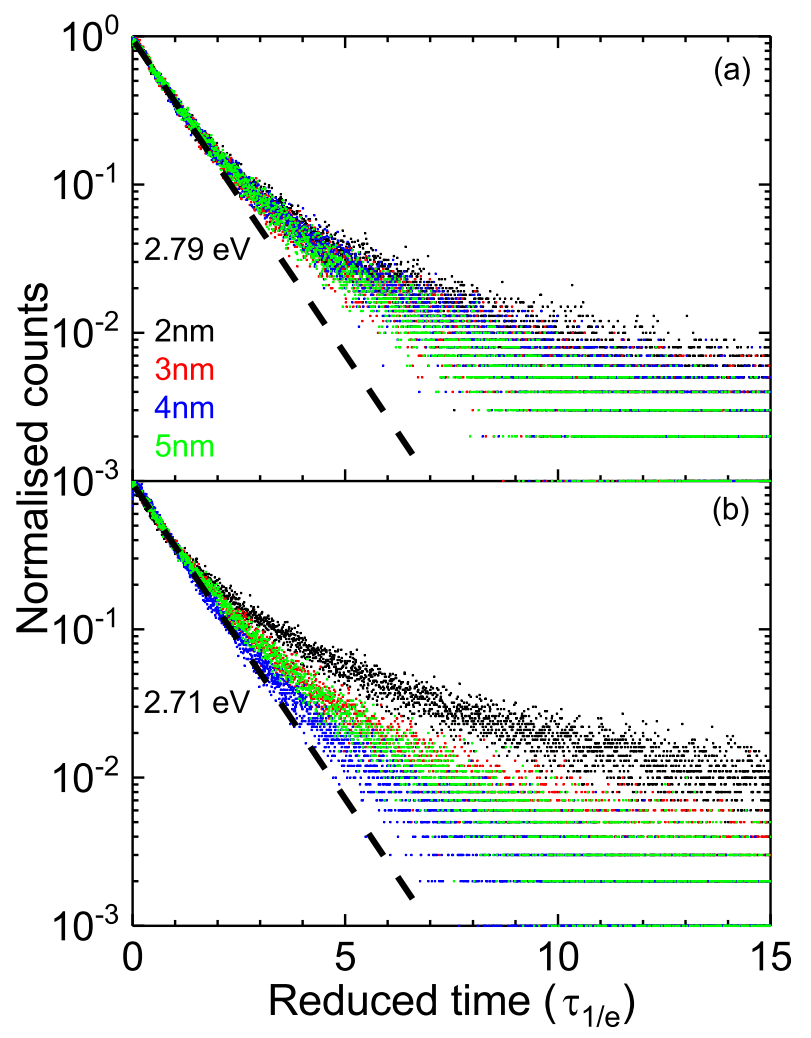

FIG. 5. $10 \mathrm{~K}$ PL decays on a reduced timescale collected at photon energies of (a) $2.79 \mathrm{eV}$ and (b) $2.71 \mathrm{eV}$.

is also reflected in the decay shapes when plotted on a reduced timescale, as shown in Fig. 5. At high energies these decay shapes are the same, but the $2 \mathrm{~nm}$-capped sample has a different decay shape at lower energies. On a reduced timescale, the decay shape has been shown to be independent of energy, provided that the localisation landscape is the same ${ }^{10}$. Therefore, these results further support the idea that the localisation differs in the $2 \mathrm{~nm}$-capped QW. This is also consistent with previous studies, which show a change in FWHM of the low temperature emission for this sample ${ }^{15}$. The change in localisation is attributed to a field direction across the QW for the $2 \mathrm{~nm}$-capped sample that has been reversed by the UL. In particular, this reversal affects the localisation of the electrons since the field now tends to push them towards the lower QW interface (i.e. closest to the $\mathrm{UL}$ ), rather than towards the upper interface as is the case for the other samples. (The nextnano3 simulations indicated that the field across the QW is weakly reversed for the $3 \mathrm{~nm}$-capped sample, but the similarity of its behaviour to that of the $4 \mathrm{~nm}$ - and $5 \mathrm{~nm}$-capped samples suggests that it actually remains unreversed.) The upper interface is significantly rougher than the lower ${ }^{20}$, and the associated regions of well widening on the upper interface provide a greater density of localisation centres 




FIG. 6. $10 \mathrm{~K}$ PLE spectra of the $2 \mathrm{~nm}$ and $5 \mathrm{~nm}$-capped QWs taken at the peak emission energy of the QW, a PL spectrum of the $2 \mathrm{~nm}$-capped QW is provided for comparison.

for electrons ${ }^{21}$. In contrast, holes are instead localised at indium fluctuations ${ }^{21}$ and so are not directly affected by the reversal of the field direction.

The decay times, and also the decay shapes, are unaffected when exciting above and below the barrier, indicating that the recombination mechanism is the same in both cases. The differences in measured IQE is therefore attributed to a process occurring before recombination. An indication of this process can be seen in the low temperature PLE measurements in Fig.6. When the QW emission is being detected, a drop in the intensity is observed if the excitation energy is reduced below $3.3 \mathrm{eV}$, which is the bandgap of the UL, as illustrated in Fig. 2. Note that the peak of the UL emission occurs at $3.19 \mathrm{eV}$, i.e. an energy less than the bandgap, because recombination chiefly involves carriers occupying localised states, which produces a redshift from the band edge ${ }^{9}$. An absorption edge from the GaN is also observed at $3.5 \mathrm{eV}^{22}$.

These spectra show that, at low temperature, carriers generated in the UL result in extra carriers in the QW. Two mechanisms for this are possible. Firstly, as the QW has a smaller bandgap than the UL, it could be due to re-absorption of the emission from the UL. This would require that carriers are captured by the UL and the subsequent emission is directly absorbed by the QW. Since the intensity of the light emitted from the UL must be less than the excitation source, this process will result in fewer carriers added to the QW in comparison to the direct absorption of the excitation light in the QW. The UL absorption edge will therefore be smaller than the direct QW absorption edge. However, this is not the case and the observed UL absorption edge is up to three times larger than the direct absorption into the QW. The second option is direct carrier transfer from the UL to the QW. As shown in Fig. 1. and in the S.M., the surface band bending results in strong electric fields in the top few $\mathrm{nm}$ of the UL. This field is sufficient to remove holes from shallow carrier traps and acts in the right direction to subsequently inject the holes into the QW. As this region is significantly wider than the QW itself, it could feasibly result in an UL absorption edge that is larger than the QW absorption edge, as is observed in Fig.6. The carrier transfer from the GaN and UL into the QW is stronger for the $2 \mathrm{~nm}$-capped QW, as evidenced by the greater intensity of the PLE edge corresponding to absorption by the UL for this sample, which is attributed to the stronger electric fields in this sample.

Further evidence for this effect can be seen in the PL time decays at short timescales. As shown in Fig.7, when detecting the laser excitation, it takes $300 \mathrm{ps}$ for the count rate to reach the peak value. This is the due to the response time of the MCP detector. When detecting the UL emission, it takes an additional $85 \mathrm{ps}$ for the peak count rate to be achieved. This difference in rise time is attributed to the time taken for photogenerated "hot" carriers to cool to the ground state before recombining ${ }^{23}$. After the maximum counts are reached, the signal decays away. When detecting the QW emission the rise time is extended by 375 ps when compared with the system response. Moreover, after reaching the peak count rate, the transient remains constant for an additional $600 \mathrm{ps}$ before decaying. This flat region of the QW decay corresponds to the 1 /e decay time of the UL, and therefore occurs only when the carrier density in the UL is depleting rapidly. This result is similar for all of the samples (see S.M.) and is further evidence of carrier transfer from the UL to the QW. The UL decay is well described by a biexponential and incorporating this into an analysis of the QW dynamics reproduces the initial rise and plateau observed (see S.M.), as shown in Fig. 7.

As also shown in the S.M., the shape of UL decay depends upon energy on a reduced timescale. As mentioned previously, this suggests that the decay does not result from recombination across a common localisation landscape. One interpretation of this result is that there is an additional energy-dependent carrier loss mechanism, such as carrier transfer from the UL to the QW, that will preferentially affect recombination in the parts of the UL adjacent to the QW.

Overall, these measurements suggests that the UL acts like a hole reservoir feeding the QWs, analogous to the electroluminescence results reported previously for MQW structures with an electron reservoir layer ${ }^{16}$. As the temperature is increased, the carrier transfer rate from the UL to the QW will increase due to the thermal escape of holes from shallow traps in the UL. This will lead to an increased density of carriers in the QW, and cause the intensity of the QW emission to be higher than if the transfer was not occurring. This can be seen qualitatively in Fig. 3: when the temperature is sufficient to reduce the UL emission intensity, the QW emission intensity increases relative to the direct excitation result. This does not represent an increase in the IQE, but rather an in- 


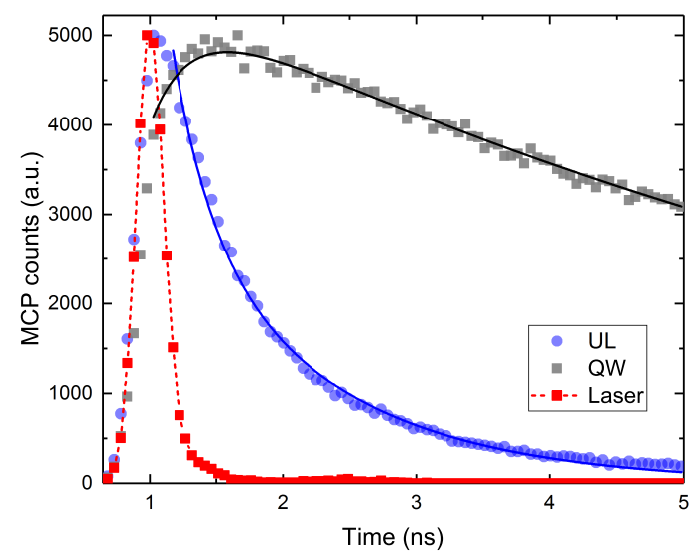

FIG. 7. $10 \mathrm{~K} \mathrm{PL}$ time decays of the $2 \mathrm{~nm}$-capped QW sample, measured with above-barrier excitation. The decays at the peak emission energy of the QW and UL are shown, along with the detected signal from the laser. The solid lines are fits to the UL and QW decays based on the rate equation analysis detailed in the S.M.

crease in the carrier density (provided the carrier density is low and any droop effect is negligible) in the QW due to an increased carrier transfer from the UL. The hole transfer effect is stronger for the $2 \mathrm{~nm}$-capped QW due to the stronger electric fields in the $\mathrm{UL}^{15}$, resulting in a greater enhancement of the QW emission intensity. The intensity plateaus around $100 \mathrm{~K}$; here the carrier transfer is increasing rapidly with increasing temperature, as evidenced by the rapid quenching of the UL intensity. The enhanced transfer for the $2 \mathrm{~nm}$-capped sample is sufficient to compensate for the reduced radiative recombination rate, such that the normalised intensity of the emission is similar to the other samples, which have a higher IQE.

\section{SUMMARY}

The room temperature IQE of these QWs for direct excitation was found to be small due to thermionic emission and was beyond the sensitivity limit of the measurement. However, when exciting above the bandgap of the barriers the effective IQE increases to a maximum of $12 \%$ due to carrier transfer from the UL into the QW. These carriers are likely to be holes, removed from shallow carrier traps by strong electric fields in the top few $\mathrm{nm}$ of the UL and subsequently injected into the QW. PLE and time decay measurements show that this occurs even at low temperatures, likely due to the surface fields which penetrate down to the UL. At elevated temperatures the carrier transfer rate increases due to thermal escape of holes from shallow traps in the UL. As the electric fields are stronger in the $2 \mathrm{~nm}$-capped QW, this resulted in a faster rate of hole transfer, which was able to compensate for the reduced room temperature IQE, resulting in an emission intensity at room temperature similar to the samples with a higher radiative recombination rate. The $\mathrm{PL}$ decay times were approximately $5 \mathrm{~ns}$ and the decay shapes were non-exponential, for all samples including those calculated to have near-zero average field across the QW. This is different from non-polar QWs, which have decay times an order of magnitude shorter and monoexponential decays, and is attributed to significant local fluctuations in the electric field ${ }^{15}$ even within samples with average electric field near zero.These results indicate that the benefit of ULs for LEDs, aside from any gettering effect ${ }^{14}$, is that, rather than reducing the radiative recombination lifetime significantly by decreasing the field across the QW, they instead act as a hole reservoir for the QWs, similar to the electron reservoirs demonstrated previously ${ }^{16}$.

${ }^{1}$ S. F. Chichibu, A. C. Abare, M. S. Minsky, S. Keller, S. B. Fleischer, J. E. Bowers, E. Hu, U. K. Mishra, L. A. Coldren, S. P. DenBaars, and T. Sota, Applied Physics Letters 73, 2006 (1998).

${ }^{2}$ F. Bernardini and V. Fiorentini, Phys. status solidi (b) 216, 391 (1999).

${ }^{3}$ S. Schulz, D. P. Tanner, E. P. O'Reilly, M. A. Caro, T. L. Martin, P. A. J. Bagot, M. P. Moody, F. Tang, J. T. Griffiths, F. Oehler, M. J. Kappers, R. A. Oliver, C. J. Humphreys, D. Sutherland, M. J. Davies, and P. Dawson, Phys. Rev. B 92, 235419 (2015).

${ }^{4}$ V. Fiorentini, F. Bernardini, F. Della Sala, A. Di Carlo, and P. Lugli, Phys. Rev. B 60, 8849 (1999).

${ }^{5}$ M. Auf der Maur, A. Pecchia, G. Penazzi, W. Rodrigues, and A. Di Carlo, Phys. Rev. Lett. 116, 027401 (2016).

${ }^{6}$ K. P. O'Donnell, M. Auf der Maur, A. Di Carlo, K. Lorenz, and the SORBET consortium, Phys. Status Solidi RRL 6, 49 (2012).

${ }^{7}$ P. Dawson, S. Schulz, R. A. Oliver, M. J. Kappers, and C. J. Humphreys, J. Appl. Phys. 119, 181505 (2016).

${ }^{8}$ S. Chichibu, K. Wada, and S. Nakamura, Appl. Phys. Lett. 71, 2346 (1997).

${ }^{9}$ O. Rubel, M. Galluppi, S. D. Baranovskii, K. Volz, L. Geelhaar, H. Riechert, P. Thomas, and W. Stolz, J. Appl. Phys. 98, 063518 (2005).

${ }^{10}$ A. Morel, P. Lefebvre, S. Kalliakos, T. Taliercio, T. Bretagnon, and B. Gil, Phys. Rev. B 68, 045331 (2003).

${ }^{11}$ M. J. Davies, S. Hammersley, F. C. Massabuau, P. Dawson, R. A. Oliver, M. J. Kappers, and C. J. Humphreys, J. Appl. Phys. 119, 055708 (2016).

${ }^{12}$ G. M. Christian, S. Hammersley, M. J. Davies, P. Dawson, M. J. Kappers, F. C.-P. Massabuau, R. A. Oliver, and C. J. Humphreys, Phys. Status Solidi C 13, 248 (2016).

${ }^{13}$ C. Haller, J. F. Carlin, G. Jacopin, D. Martin, R. Butté, and N. Grandjean, Appl. Phys. Lett. 111 (2017).

${ }^{14}$ C. Haller, J.-F. Carlin, M. Mosca, M. D. Rossell, R. Erni, and N. Grandjean, Appl. Phys. Express 12, 034002 (2019).

${ }^{15}$ G. M. Christian, S. Schulz, S. Hammersley, M. J. Kappers, M. Frentrup, C. J. Humphreys, R. A. Oliver, and P. Dawson, Jpn. J. Appl. Phys. 58, SCCB09 (2019).

${ }^{16}$ N. Otsuji, K. Fujiwara, and J. K. Sheu, Journal of Applied Physics 100, 113105 (2006).

${ }^{17}$ D. M. Graham, A. Soltani-Vala, P. Dawson, M. J. Godfrey, T. M. Smeeton, J. S. Barnard, M. J. Kappers, C. J. Humphreys, and E. J. Thrush, J. Appl. Phys. 97, 103508 (2005).

${ }^{18}$ P. Hurst, P. Dawson, S. Levetas, M. Godfrey, I. Watson, and G. Duggan, Phys. status solidi 228, 137 (2001).

${ }^{19}$ W. Shan, X. C. Xie, J. J. Song, and B. Goldenberg, Appl. Phys. Lett. 67, 2512 (1995). 
${ }^{20}$ M. J. Galtrey, R. A. Oliver, M. J. Kappers, C. J. Humphreys, P. H. Clifton, D. Larson, D. W. Saxey, and A. Cerezo, Journal of Applied Physics 104, 013524 (2008).

${ }^{21}$ D. Watson-Parris, M. J. Godfrey, P. Dawson, R. a. Oliver, M. J. Galtrey, M. J. Kappers, and C. J. Humphreys, Phys. Rev. B 83, 115321 (2011).

${ }^{22}$ I. Vurgaftman and J. R. Meyer, J. Appl. Phys. 94, 3675 (2003).

${ }^{23}$ S. Boubanga-tombet, J. B. Wright, P. Lu, M. R. C. Williams, C. Li, G. T. Wang, and R. P. Prasankumar, ACS Photonics 3, 2237 (2016)

\section{SUPPLEMENTARY MATERIAL}

See the supplementary material for the simulated $\mathrm{CB}$ and VB profiles for all samples; the temperature- dependence of intensity for all samples; the UL time decays on a reduced timescale; the QW time decays on short timescales; and details of the equations used to fit the QW and UL time decays in Fig. 7.

\section{ACKNOWLEDGMENTS}

This was work funded by EPSRC under grants $\mathrm{EP} / \mathrm{M} 010627 / 1$ and EP/M010589/1. Research data supporting this publication are available at DOI: $10.48420 / 14754504$. 